\title{
Acute toxicity of lethal and sublethal concentrations of neonicotinoid, insect growth regulator and diamide insecticides on natural enemies of the woolly apple aphid and the obscure mealybug
}

Catalina Radrigán-Navarro $^{{ }^{*}}$, Elizabeth H. Beers ${ }^{2}$, Andrés Alvear ${ }^{3}$, and Eduardo Fuentes-Contreras ${ }^{1}$

'Universidad de Talca, Facultad de Ciencias Agrarias, Casilla 747, Talca, Chile. ${ }^{*}$ Corresponding author (cradrigan@utalca.cl).

${ }^{2}$ Washington State University, Tree Fruit Extension Centre, 1100 N Western Ave., Wenatchee, Washington, USA.

${ }^{3}$ Xilema SpA Departamento de Investigación y Desarrollo, Ruta 64 km 2.5, Quillota, Chile.

Received: 1 February 2021; Accepted: 3 May 2021; doi:10.4067/S0718-58392021000300398

\begin{abstract}
Based on the importance of using low-risk compounds to protect beneficial agents, the present study evaluated the acute toxicity of lethal and sublethal concentrations of the insecticides thiacloprid, pyriproxyfen, and chlorantraniliprole on adults of Aphelinus mali (Haldeman), the main parasitoid of the woolly apple aphid, Eriosoma lanigerum (Hausmann). Similarly, acetamiprid, buprofezin, pyriproxyfen, chlorantraniliprole and cyantraniliprole were evaluated on adults of the parasitoid Acerophagus flavidulus (Brethés) and also on larvae and adults of the predator Cryptolaemus montrouzieri Mulsant, both species important natural enemies of the obscure mealybug, Pseudococcus viburni (Signoret). Natural enemies were exposed to insecticide residues of minimum recommended rate $(1 \mathrm{x})$ and between one to four concentrations $(0.5 \mathrm{x}, 0.25 \mathrm{x}, 0.1 \mathrm{x}$ and $0.05 \mathrm{x})$ of the minimum recommended rate on apple leaves. Our results indicate that thiacloprid was moderately harmful to A. mali at $1 \mathrm{x}$, and acetamiprid was harmful to A. flavidulus causing $100 \%$ mortality from $0.1 \mathrm{x}$ to $1 \mathrm{x}$ concentrations. Buprofezin, pyriproxyfen and chlorantraniliprole were harmless or slightly harmful for both parasitoids. Cyantraniliprole was slightly harmful from $0.25 \mathrm{x}$ to $1 \mathrm{x}$ for A. flavidulus. For C. montrouzieri, acetamiprid was harmless or slightly harmful for larvae and adults on the concentrations evaluated. Buprofezin, pyriproxyfen and chlorantraniliprole were harmless for larvae and adults of $C$. montrouzieri. Cyantraniliprole was slightly harmful on larvae and moderately harmful on adults of $C$. montrouzieri at 1x. Our data suggest that buprofezin, pyriproxyfen and chlorantraniliprole could be compatible with the natural enemies evaluated, while thiacloprid and acetamiprid were less compatible with parasitoids than with the predator. Finally, cyantraniliprole seems to be less compatible than chlorantraniliprole at $1 \mathrm{x}$ with the natural enemies evaluated.
\end{abstract}

Key words: Aphelinidae, Coccinellidae, ecotoxicology, Encyrtidae, integrated pest management, mortality.

\section{INTRODUCTION}

Apple (Malus domestica (Suckow) Borkh.) is an important fruit crop in many regions around the world, including central Chile and the West Coast of USA. Both regions share pest problems among which phloem-feeding pests, such as the woolly apple aphid (WAA), Eriosoma lanigerum (Hausmann) (Hemiptera: Aphididae), and mealybugs, Pseudococcus spp. (Hemiptera: Pseudococcidae), are important secondary pests. In apple orchards frequent applications of broadspectrum insecticides can be the key factor explaining the limited success of some natural enemies, and the use of more selective insecticides is an important strategy to improve the integration of chemical and conservation biological control (Jones et al., 2009; Weddle et al., 2009). 
Insecticides used to control codling moth (CM), Cydia pomonella (L.) (Lepidoptera: Tortricidae), and other pests in apple orchards can disrupt biological control (Beers et al., 2016). Some of these are wide-spectrum neurotoxic insecticides, such as the neonicotinoids thiacloprid and acetamiprid (Casida, 2018) while other are more selective insect growth regulators (IGRs), such as buprofezin and pyriproxyfen (Tunaz and Uygun, 2004) or newer muscular contraction disruptors such as the anthranilinic diamides chlorantraniliprole and cyantraniliprole (Lahm et al., 2005; 2007; Selby et al., 2013; Ahumada and Chorbadjian, 2019).

Among the natural enemies of phloem-feeding pests, Aphelinus mali (Haldeman) (Hymenoptera: Aphelinidae) is the most important parasitoid of the WAA around the world. Aphelinus mali is a specialist, solitary, koinobiont endoparasitoid successfully introduced to many countries for the control of WAA (Howard, 1929; Asante, 1997; Cross et al., 1999). Regarding the natural enemies of the obscure mealybug (OMB), Pseudococcus viburni (Signoret) (Hemiptera: Pseudococcidae), in Chile the parasitoid Acerophagus flavidulus (Brethés) (Hymenoptera: Encyrtidae) is the most relevant (González, 2010; Charles, 2011). Acerophagus flavidulus is a specialist, gregarious, koinobiont endoparasitoid native to Argentina and Chile (Karamaouna and Copland, 2000; Noyes, 2003). On the other hand, the predator Cryptolaemus montrouzieri Mulsant (Coleoptera: Coccinellidae) is native to Australia and has been used in augmentative releases against mealybugs in many crops and countries (Kairo et al., 2013; Mani, 2018).

The aim of the study was to provide the necessary data to build integrated pest management programs of pome fruit orchards. We assess the acute toxicity of lethal (minimum field recommended rate) and sublethal (dilutions from minimum field recommended rate) concentrations of six insecticides frequently used for the main apple pests in pome fruit orchards in Chile, on adults of the parasitoids A. mali and A. flavidulus, and also on larvae and adults of the predator C.montrouzieri. We exposed parasitoids and predator to insecticide residues on apple leaves under laboratory conditions, in order to evaluate mortality effect of insecticide concentrations on these natural enemies.

\section{MATERIALS AND METHODS}

\section{Insect material}

The parasitoids Aphelinus mali (Haldeman) were collected as mummies from organic apple (Malus domestica (Suckow) Borkh.) trees of the Sunrise Research Farm, Tree Fruit Research and Extension Center, Washington State University (WSU), Wenatchee, USA. The parasitoid mummies were carefully placed in $30 \mathrm{~mL}$ plastic cups covered with a plastic lid. These cups were maintained in a growth room with a controlled environment $\left(25 \pm 1{ }^{\circ} \mathrm{C}, 40 \pm 5 \% \mathrm{RH}\right.$ and $16: 8 \mathrm{~h}$ photoperiod) until the emergence of the adult parasitoid. The parasitoid Acerophagus flavidulus (Brethés) and the predator Cryptolaemus montrouzieri Mulsant were reared at Xilema SpA, a biological control company located in Quillota, Chile. Parasitoid mummies, predator larvae and newly eclosed adults were sent weekly to laboratories of Sanidad Vegetal at Universidad de Talca (UTAL), Talca, Chile. The A. flavidulus mummies were kept in a growth chamber (Conviron, Winnipeg, Canada) at $25 \pm 0.1{ }^{\circ} \mathrm{C}, 50 \pm 5 \% \mathrm{RH}$ and a photoperiod of $16: 8 \mathrm{~h}$ until adult emergence (usually 2 to $5 \mathrm{~d}$ after laboratory arrival).

\section{Application of insecticides}

Aphelinus mali was exposed to 96.0 and $9.6 \mathrm{mg} \mathrm{L}^{-1}$ ai thiacloprid ([3-[(6-chloropyridin-3-yl)methyl]-1,3-thiazolidin-2ylidene]cyanamide), 50 and $5 \mathrm{mg} \mathrm{L}^{-1}$ ai pyriproxyfen (2-[1-(4-phenoxyphenoxy)propan-2-yloxy]pyridine) and 40 and $4 \mathrm{mg} \mathrm{L}^{-1}$ ai chlorantraniliprole (5-bromo-N-[4-chloro-2-methyl-6-(methylcarbamoyl)phenyl]-2-(3-chloropyridin-2-yl) pyrazole-3-carboxamide), equivalent to the minimum recommended rate $(1 \mathrm{x})$ and one tenth of that concentration $(0.1 \mathrm{x})$ for codling moth (CM) (Cydia pomonella (L.); Lepidoptera: Tortricidae), woolly apple aphid (WAA) (Eriosoma lanigerum (Hausmann); Hemiptera: Aphididae) and obscure mealybug (OMB) (Pseudococcus viburni (Signoret); Hemiptera: Pseudococcidae) on apples (Table 1). Acerophagus flavidulus and C. montrouzieri were exposed to acetamiprid ( $N-[(6-$ chloropyridin-3-yl)methyl]- $N$ '-cyano- $N$-methylethanimidamide $N$ ), buprofezin (2-tert-butylimino-5-phenyl-3-propan-2yl-1,3,5-thiadiazinan-4-one), pyriproxyfen, chlorantraniliprole, and cyantraniliprole (5-bromo-2-(3-chloropyridin-2-yl)$N$-[4-cyano-2-methyl-6-(methylcarbamoyl)phenyl]pyrazole-3-carboxamide) (Table 1). 
Table 1. Insecticides and concentrations applied on apple leaf discs in toxicity test for natural enemies of the woolly apple aphid and the obscure mealybug.

\begin{tabular}{lllc}
\hline $\begin{array}{l}\text { Commercial } \\
\text { products (cp) }\end{array}$ & \multicolumn{1}{c}{ Active ingredient (chemical group) } & \multicolumn{1}{c}{ Manufacturer } & $\begin{array}{c}\text { Minimum } \\
\text { recommended } \\
\text { cp rate }\end{array}$ \\
\hline Calypso 480 SC & Thiacloprid (neonicotinoid) & Bayer, AG, Germany & $\begin{array}{c}\mathrm{mL} \mathrm{or} \mathrm{g} \mathrm{L}^{-1} \\
0.2 \mathrm{~mL}\end{array}$ \\
Hurricane 70 WP & Acetamiprid (neonicotinoid) & Anasac SpA, Chile & $0.12 \mathrm{~g}$ \\
Applaud 25 WP & Buprofezin (thiadiazine) & Anasac SpA, Chile & $0.8 \mathrm{~g}$ \\
Delico 100 EC & Pyriproxyfen (pyridine) & Anasac SpA, Chile & $0.5 \mathrm{~mL}$ \\
Coragen 20 SC & Chlorantraniliprole (anthranilic diamide) & FMC, USA & $0.2 \mathrm{~mL}$ \\
Exirel 10 SE & Cyantraniliprole (anthranilic diamide) & FMC, USA & $0.75 \mathrm{~mL}$ \\
\hline
\end{tabular}

Both OMB natural enemies were exposed to $84.00,42.00,21.00,8.40$ and $0.42 \mathrm{mg} \mathrm{L}^{-1}$ ai acetamiprid; 200, 100, 50, 20 and $10 \mathrm{mg} \mathrm{L}^{-1}$ ai buprofezin; 50.0, 25.0, 12.5, 5.0 and $2.5 \mathrm{mg} \mathrm{L}^{-1}$ ai pyriproxyfen; 40, 20, 10, 4 and $2 \mathrm{mg} \mathrm{L}^{-1}$ ai chlorantraniliprole and $75.00,37.50,18.75,7.50$ and $3.75 \mathrm{mg} \mathrm{L}^{-1}$ ai cyantraniliprole. Each active ingredient concentration mentioned was equivalent to $1 \mathrm{x}, 0.50 \mathrm{x}, 0.25 \mathrm{x}, 0.10 \mathrm{x}$ and $0.05 \mathrm{x}$ of the minimum rate recommended for $\mathrm{CM}$, WAA or OMB on apples. These concentrations were used to represent the degradation of insecticides residues over time. A volume of 2 $\mathrm{mL}$ of each insecticide concentration was applied at $0.045 \mathrm{MPa}$ with a Potter precision laboratory spray tower (Burkard Scientific, Uxbridge, UK) on an apple leaf disk with the lower surface facing up on a pad of moist cotton inside a $30 \mathrm{~mL}$ plastic cup (Beers et al., 2009). Distilled water was used as control.

\section{Bioassays}

The same methodology of exposure to dry insecticide residues on apple leaves was used for the three natural enemies as described by Gontijo (2011). The bioassay arena consisted in a $30 \mathrm{~mL}$ plastic cup with which had a lid with a small orifice $(\approx$ $5 \mathrm{~mm}$ in diameter) on the top covered by Micropore tape (3M, St. Paul, Minnesota, USA). Inside the cup, an apple leaf disk of $40 \mathrm{~mm}$ diameter was sprayed with insecticides or distilled water. Once the treated surface dried, one adult or larva of each natural enemy was placed over the leaf. All plastic cups were maintained in a Conviron chamber at $25 \pm 0.1^{\circ} \mathrm{C}, 50 \pm 5 \% \mathrm{RH}$ and a photoperiod of 16:8 h. Parasitoid adults which were 2-3 d old were used in the experiments in the WSU and UTAL laboratories. Cryptolaemus montrouzieri second-third instar larvae and newly eclosed adults were used on the day of arrival to the UTAL laboratories. Natural enemies were not fed during the exposure period. Evaluation of mortality was performed after 24 and $48 \mathrm{~h}$ exposure. Parasitoids and predators were considered dead if they did not move when probed with a fine brush (Mgocheki and Addison, 2009; Rogers et al., 2011; Wang et al., 2019). The experimental unit was an individual natural enemy. For each treatment, between 12-30 experimental units were used simultaneously. These whole bioassays were replicated three times.

\section{Statistical analysis}

Data were analyzed as categorical with binary response (dead or alive) using a general linear model (GLM) with binomial distribution (R software; R Foundation for Statistical Computing, Vienna, Austria.) in order to compare different concentrations within each tested insecticide. Results were reported and analyzed only when control mortality was $\leq 30 \%$. Significant differences $(\mathrm{P} \leq 0.05)$ between treatments were evaluated with Tukey test using the "multcomp" R package. Mortality data were corrected using Abbott's formula and negative values are shown as zero when presenting data in the tables (Abbott, 1925). 
Table 2. Mortality of Aphelinus mali at 24 and $48 \mathrm{~h}$ after exposure to insecticide treatments on apple leaf discs.

\begin{tabular}{|c|c|c|c|c|c|c|}
\hline \multirow[b]{2}{*}{ Insecticide } & \multirow{2}{*}{$\begin{array}{l}\text { Active ingredient } \\
\text { concentration }\end{array}$} & \multirow{2}{*}{$\begin{array}{l}\text { Commercial } \\
\text { product rate }\end{array}$} & \multirow[b]{2}{*}{$\mathrm{n}$} & \multicolumn{2}{|c|}{ Corrected mortality } & \multirow{2}{*}{$\begin{array}{l}\text { IOBC } \\
\text { class }\end{array}$} \\
\hline & & & & $24 \mathrm{~h}$ & $48 \mathrm{~h}$ & \\
\hline & $\mathrm{mg} \mathrm{L}^{-1}$ & & & $\%$ & $\%$ & \\
\hline \multirow{4}{*}{ Thiacloprid } & 0 & Control & 30 & $15.0 \pm 10.6 \mathrm{a}$ & $26.7 \pm 18.8 \mathrm{a}$ & \\
\hline & 9.6 & $0.1 \mathrm{x}$ & 30 & $43.1 \pm 15.0 \mathrm{~b}$ & $45.5 \pm 13.3 b$ & 2 \\
\hline & 96.0 & $1 \mathrm{x}$ & 30 & $84.3 \pm 6.7 \mathrm{c}$ & $93.2 \pm 1.7 \mathrm{c}$ & 3 \\
\hline & & $\chi^{2}, P$ & & $68.49 \leq 0.01$ & $67.28 \leq 0.01$ & \\
\hline \multirow[t]{4}{*}{ Pyriproxyfen } & 0 & Control & 30 & $10.0 \pm 3.3 \mathrm{a}$ & $10.0 \pm 3.3 \mathrm{a}$ & \\
\hline & 5 & $0.1 \mathrm{x}$ & 30 & $3.7 \pm 0.0 \mathrm{a}$ & $7.4 \pm 3.3 \mathrm{a}$ & 1 \\
\hline & 50 & $1 \mathrm{x}$ & 30 & $63.0 \pm 6.7 \mathrm{~b}$ & $70.4 \pm 0.0 \mathrm{~b}$ & 2 \\
\hline & & $\chi^{2}, P$ & & $28.69 \leq 0.01$ & $33.24 \leq 0.01$ & \\
\hline \multirow{4}{*}{ Chlorantraniliprole } & 0 & Control & 34 & 0 & 0 & \\
\hline & 4 & $0.1 \mathrm{x}$ & 27 & 0 & 0 & \\
\hline & 40 & $1 \mathrm{x}$ & 29 & 0 & $3.5 \pm 0.2$ & 1 \\
\hline & & $\chi^{2}, P$ & & $0 \geq 0.05$ & $2.29 \geq 0.05$ & 1 \\
\hline
\end{tabular}

Values within columns followed by a different letter are significantly different according to Tukey's test ( $\mathrm{P} \leq 0.05)$. Values are mean $\pm \mathrm{SE}$; n: number of insects tested. Control non corrected by Abbott's formula.

IOBC class: 1: Harmless (mortality < 30\%), 2: slightly harmful $(30 \leq$ mortality $\leq 79 \%), 3$ : moderately harmful $(80 \leq$ mortality $\leq 99 \%)$, and 4: harmful (mortality $>99 \%$ ).

\section{RESULTS}

The mortality of adult A. mali was significantly increased with the minimum recommended $\left(96 \mathrm{mg} \mathrm{L}^{-1}\right)$ and $9.6 \mathrm{mg} \mathrm{L}^{-1}$ concentrations of ai thiacloprid in comparison with the control after 24 and $48 \mathrm{~h}$ (Table 2). Pyriproxyfen applied at the lowest concentration $\left(5 \mathrm{mg} \mathrm{L}^{-1}\right)$ showed no difference in mortality with the control, but at the minimum recommended rate $\left(50 \mathrm{mg} \mathrm{L}^{-1}\right)$ mortality increased significantly for 24 and $48 \mathrm{~h}$. On the contrary, chlorantraniliprole did not increase adult parasitoid mortality of A. mali in both concentrations evaluated.

From the lowest concentration $\left(0.42 \mathrm{mg} \mathrm{L}^{-1}\right)$ to the minimum recommended rate $\left(84 \mathrm{mg} \mathrm{L}^{-1}\right)$, acetamiprid significantly increased adult mortality of A. flavidulus in relation with the control treatment (Table 3). For all concentrations of insect growth regulators (IGRs) buprofezin and pyriproxyfen tested, adult mortality of A. flavidulus was not significantly different from control treatment. For chlorantraniliprole, only $0.25 \mathrm{x}$ concentration $\left(10 \mathrm{mg} \mathrm{L}^{-1}\right)$ was significantly higher than control. For cyantraniliprole all treatments except for the two lowest ones ( 3.75 and $7.5 \mathrm{mg} \mathrm{L}^{-1}$ ) were significantly higher than control (Table 3).

Higher mortality than control treatment was found for larvae of $C$. montrouzieri exposed to 0.5 and $1 \mathrm{x}$ rates of acetamiprid at $24 \mathrm{~h}$, equivalent to 42 and $84 \mathrm{mg} \mathrm{L}^{-1}$, respectively (Table 4). Larvae of C. montrouzieri exposed to all concentrations tested of buprofezin and pyriproxyfen, showed nonsignificant differences in mortality from the control treatment at $48 \mathrm{~h}$. The same was observed for chlorantraniliprole at $24 \mathrm{~h}$. Only the minimum recommended rate of cyantraniliprole at $48 \mathrm{~h}$ significantly increased larval mortality relative to the control (Table 4).

Similar results were obtained for adults of $C$. montrouzieri, with neither of the IGRs (all concentrations) causing a significant level of mortality relative to the control (Table 5). For chlorantraniliprole, only 10 and $40 \mathrm{mg} \mathrm{L}^{-1}$ significantly increased adult mortality at $48 \mathrm{~h}$. Acetamiprid significantly increased adult mortality at all concentrations tested at $48 \mathrm{~h}$. Similarly, cyantraniliprole at all concentrations over $18.75 \mathrm{mg} \mathrm{L}^{-1}$ significantly increased adult mortality relative to the control at $48 \mathrm{~h}$ (Table 5).

\section{DISCUSSION}

The International Organization for Biological Control (IOBC) recommends a tiered approach to evaluate insecticide compatibility with natural enemies, whereby insecticide testing is performed in the laboratory and depending upon the results obtained, semi-field or field tests may be included. The IOBC classifies pesticides into four categories depending on the extent of mortality or reduction in life history performance that they cause to natural enemies: harmless (class 1), slightly harmful (class 2), moderately harmful (class 3 ) and harmful (class 4), which correspond to reductions below $30 \%$, between $31 \%$ and $79 \%$, between $80 \%$ and $99 \%$ and higher than $99 \%$, respectively (Sterk et al., 1999). 
Table 3. Mortality of adult Acerophagus flavidulus at $24 \mathrm{~h}$ after exposure to insecticide treatments on apple leaf discs.

\begin{tabular}{|c|c|c|c|c|c|}
\hline Insecticide & $\begin{array}{l}\text { Active ingredient } \\
\text { concentration }\end{array}$ & $\begin{array}{l}\text { Commercial } \\
\text { product rate }\end{array}$ & $\mathrm{n}$ & Corrected mortality, $24 \mathrm{~h}$ & $\begin{array}{l}\text { IOBC } \\
\text { class }\end{array}$ \\
\hline & $\mathrm{mg} \mathrm{L}^{-1}$ & & & $\%$ & \\
\hline \multirow{7}{*}{ Acetamiprid } & 0 & Control & 12 & $27.8 \pm 6.2 \mathrm{a}$ & \\
\hline & 0.42 & $0.05 x$ & 10 & $65.4 \pm 6.2 \mathrm{~b}$ & 2 \\
\hline & 8.40 & $0.10 \mathrm{x}$ & 12 & $100.0 \pm 0.0 \mathrm{~b}$ & 4 \\
\hline & 21.00 & $0.25 \mathrm{x}$ & 12 & $100.0 \pm 0.0 \mathrm{~b}$ & 4 \\
\hline & 42.00 & $0.50 \mathrm{x}$ & 12 & $100.0 \pm 0.0 \mathrm{~b}$ & 4 \\
\hline & 84.00 & $1 \mathrm{x}$ & 12 & $100.0 \pm 0.0 \mathrm{~b}$ & 4 \\
\hline & & $\chi^{2}, \mathrm{P}$ & & $93.48 \leq 0.01$ & \\
\hline \multirow[t]{7}{*}{ Buprofezin } & 0 & Control & 27 & $14.8 \pm 6.4$ & \\
\hline & 10 & $0.05 x$ & - & - & \\
\hline & 20 & $0.10 \mathrm{x}$ & 13 & $0.6 \pm 7.3$ & 1 \\
\hline & 50 & $0.25 \mathrm{x}$ & 13 & $0.4 \pm 8.7$ & 1 \\
\hline & 100 & $0.50 \mathrm{x}$ & 13 & $1.7 \pm 6.4$ & 1 \\
\hline & 200 & $1 \mathrm{x}$ & 13 & $0.3 \pm 8.8$ & 1 \\
\hline & & $\chi^{2}, \mathrm{P}$ & & $0.26 \geq 0.05$ & \\
\hline \multirow{7}{*}{ Pyriproxyfen } & 0 & Control & 18 & $23.7 \pm 8.8$ & \\
\hline & 2.5 & $0.05 x$ & - & - & \\
\hline & 5.0 & $0.10 \mathrm{x}$ & 13 & $6.4 \pm 10.2$ & 1 \\
\hline & 12.5 & $0.25 \mathrm{x}$ & 13 & $1.4 \pm 16.2$ & 1 \\
\hline & 25.0 & $0.50 \mathrm{x}$ & 13 & $16.3 \pm 10.8$ & 1 \\
\hline & 50.0 & $1 \mathrm{x}$ & 13 & $18.4 \pm 12.2$ & 1 \\
\hline & & $\chi^{2}, \mathrm{P}$ & & $3.46 \geq 0.05$ & \\
\hline \multirow[t]{7}{*}{ Chlorantraniliprole } & 0 & Control & 12 & $3.3 \pm 3.3 \mathrm{a}$ & \\
\hline & 2 & $0.05 \mathrm{x}$ & 12 & $6.9 \pm 1.4 \mathrm{a}$ & 1 \\
\hline & 4 & $0.10 \mathrm{x}$ & 12 & $8.3 \pm 2.9 \mathrm{a}$ & 1 \\
\hline & 10 & $0.25 \mathrm{x}$ & 13 & $41.2 \pm 8.4 \mathrm{~b}$ & 2 \\
\hline & 20 & $0.50 x$ & 12 & $28.4 \pm 4.6 \mathrm{a}$ & 1 \\
\hline & 40 & $1 \mathrm{x}$ & 13 & $23.5 \pm 5.9 \mathrm{a}$ & 1 \\
\hline & & $\chi^{2}, P$ & & $33.06 \leq 0.01$ & \\
\hline \multirow[t]{7}{*}{ Cyantraniliprole } & 0 & Control & 12 & $11.7 \pm 7.3 \mathrm{a}$ & \\
\hline & 3.75 & $0.05 x$ & 12 & $20.8 \pm 6.9 \mathrm{a}$ & 1 \\
\hline & 7.50 & $0.10 \mathrm{x}$ & 12 & $20.8 \pm 6.9 \mathrm{a}$ & 1 \\
\hline & 18.75 & $0.25 x$ & 12 & $39.0 \pm 2.4 \mathrm{~b}$ & 2 \\
\hline & 37.50 & $0.50 x$ & 12 & $47.8 \pm 2.4 \mathrm{~b}$ & 2 \\
\hline & 75.00 & $1 \mathrm{x}$ & 12 & $54.0 \pm 0.6 \mathrm{~b}$ & 2 \\
\hline & & $\chi^{2}, P$ & & $28.5 \leq 0.01$ & \\
\hline
\end{tabular}

Values within columns followed by a different letter are significantly different according to Tukey's test $(\mathrm{P} \leq 0.05)$. Values are mean $\pm \mathrm{SE}$; n: number of insects tested. Control not corrected by Abbott's formula.

IOBC class: 1: Harmless (mortality < 30\%), 2: slightly harmful $(30 \leq$ mortality $\leq 79 \%), 3$ : moderately harmful $(80 \leq$ mortality $\leq 99 \%)$, and 4: harmful (mortality $>99 \%)$.

Our results indicate that neurotoxic neonicotinoid thiacloprid was moderately harmful to A. mali at the minimum recommended rate, and acetamiprid was harmful to A. flavidulus on almost all concentrations evaluated.

IGRs were harmless at low concentrations on A. flavidulus and slightly harmful at minimum rate only on A. mali, which is associated with the mode of action on this group of insecticides on immature stages. The diamide chlorantraniliprole was harmless to both parasitoids with the exception of $10 \mathrm{mg} \mathrm{L}^{-1}$ ai, which was slightly harmful for A. flavidulus. On the other hand, the newer diamide cyantraniliprole was slightly harmful from 18.75 to $75 \mathrm{mg} \mathrm{L}^{-1}$ ai for A. flavidulus. For C. montrouzieri acetamiprid was harmless on larvae and slightly harmful for adults, suggesting a less detrimental effect on the predator than on the parasitoid of the OMB. Both IGRs and chlorantraniliprole were harmless for larvae and adults. Cyantraniliprole was slightly harmful on larvae and moderately harmful on adults of C. montrouzieri at the minimum recommended rate.

Moderately harmful results of thiacloprid on A. mali agree with results from Gontijo (2011) but differ from Rogers et al. $(2011 ; 2015)$ which found thiacloprid to be harmless on this parasitoid. In addition, Ebadollahi and Sadeghi (2020) found thiacloprid slightly harmful at $48 \mathrm{~h}$, which only occurred in our study with $0.1 \mathrm{x}$ rate of the commercial product (9.6 $\mathrm{mg} \mathrm{L}^{-1}$ ai). For other encyrtid parasitoids of mealybugs, acetamiprid was harmless at the recommended rate on 
Leptomastix dactylopii Howard, but when $4 \mathrm{x}$ the recommended rate was applied acetamiprid produced $100 \%$ mortality on this parasitoid (Cloyd and Dickinson, 2006). We found acetamiprid harmful to A. flavidulus at even low concentrations, which might be related with differences in body size between adult parasitoid species. In fact, the head width of $A$. flavidulus is between 0.26-0.29 mm (Karamaouna and Copland, 2000) while the head width of L. dactylopii is twice around 0.53-0.58 mm (Jong and van Alphen, 1989). Cloyd and Dickinson (2006) found acetamiprid harmful on adult $C$. montrouzieri, although less toxic than organophosphate insecticides in other study (Dumaniya et al., 2015). However, we found that acetamiprid was slightly harmful to adults. This could be related with methodological differences in bioassays, because Cloyd and Dickinson (2006) used direct spray on adults of $C$. montrouzieri, a different formulation (30 water soluble granules vs. 70 wettable powder) and water volume $(4 v s .2 \mathrm{~mL})$ to spray acetamiprid that resulted in a lower amount of active ingredient used in our study ( $24 \mathrm{vs} .16 \mathrm{mg}$ per experimental unit). Furthermore, in semi-field bioassays, acetamiprid residues were harmless for adults of $C$. montrouzieri $35 \mathrm{~d}$ after treatment (DAT) (Alvear et al., 2016). Foliar surface residues of acetamiprid on apple decreased to lower than 50\% 7 DAT (Jamil et al., 2019), which might result in foliar surface residues lower than $0.42 \mathrm{mg} \mathrm{L}^{-1}$ ai at 35 DAT.

Table 4. Mortality of second-third instar larvae of Cryptolaemus montrouzieri at 24 and $48 \mathrm{~h}$ after exposure to insecticide treatments on apple leaf discs.

\begin{tabular}{|c|c|c|c|c|c|c|}
\hline \multirow[b]{2}{*}{ Insecticide } & \multirow{2}{*}{$\begin{array}{l}\text { Active ingredient } \\
\text { concentration }\end{array}$} & \multirow{2}{*}{$\begin{array}{l}\text { Commercial } \\
\text { product rate }\end{array}$} & \multirow[b]{2}{*}{$\mathrm{n}$} & \multicolumn{2}{|c|}{ Corrected mortality } & \multirow{2}{*}{$\begin{array}{l}\text { IOBC } \\
\text { class }\end{array}$} \\
\hline & & & & $24 \mathrm{~h}$ & $48 \mathrm{~h}$ & \\
\hline & $\mathrm{mg} \mathrm{L}^{-1}$ & & & $\%$ & $\%$ & \\
\hline \multirow{7}{*}{ Acetamiprid } & 0 & Control & 18 & $16.8 \pm 5.7 \mathrm{a}$ & - & \\
\hline & 0.42 & $0.05 \mathrm{x}$ & - & - & - & \\
\hline & 8.40 & $0.10 \mathrm{x}$ & 18 & $0.0 \pm 3.1 \mathrm{a}$ & - & 1 \\
\hline & 21.00 & $0.25 \mathrm{x}$ & 18 & $7.4 \pm 2.7 \mathrm{ab}$ & - & 1 \\
\hline & 42.00 & $0.50 \mathrm{x}$ & 19 & $33.9 \pm 2.9 \mathrm{~b}$ & - & 2 \\
\hline & 84.00 & $1 \mathrm{x}$ & 19 & $15.4 \pm 5.4 b$ & - & 1 \\
\hline & & $\chi^{2}, \mathrm{P}$ & & $22.95 \leq 0.01$ & - & \\
\hline \multirow[t]{7}{*}{ Buprofezin } & 0 & Control & 18 & $6.7 \pm 3.9$ & $12.9 \pm 3.9$ & \\
\hline & 10 & $0.05 \mathrm{x}$ & 18 & $0.0 \pm 3.9$ & $3.3 \pm 4.2$ & 1 \\
\hline & 20 & $0.10 x$ & 15 & $11.9 \pm 8.0$ & $15.8 \pm 7.7$ & 1 \\
\hline & 50 & $0.25 x$ & 18 & $0.0 \pm 3.9$ & $8.7 \pm 0.5$ & 1 \\
\hline & 100 & $0.50 \mathrm{x}$ & 15 & $4.8 \pm 5.9$ & $10.7 \pm 5.9$ & 1 \\
\hline & 200 & $1 \mathrm{x}$ & 15 & $0.0 \pm 6.7$ & $0.0 \pm 0.0$ & 1 \\
\hline & & $\chi^{2}, \mathrm{P}$ & & $5.31 \geq 0.05$ & $6.23 \geq 0.05$ & \\
\hline \multirow{7}{*}{ Pyriproxyfen } & 0 & Control & 13 & $17.8 \pm 15$ & $22.2 \pm 13.5$ & \\
\hline & 2.5 & $0.05 x$ & 13 & $0.0 \pm 7.8$ & $0.0 \pm 2.2$ & 1 \\
\hline & 5.0 & $0.10 x$ & 13 & $0.0 \pm 8.0$ & $0.0 \pm 0.0$ & 1 \\
\hline & 12.5 & $0.25 x$ & 13 & $0.0 \pm 10.2$ & $0.0 \pm 0.0$ & 1 \\
\hline & 25.0 & $0.50 \mathrm{x}$ & 13 & $0.0 \pm 3.9$ & $0.0 \pm 0.0$ & 1 \\
\hline & 50.0 & $1 \mathrm{x}$ & 13 & $0.0 \pm 12.4$ & $2.85 \pm 13.5$ & 1 \\
\hline & & $\chi^{2}, \mathrm{P}$ & & $3.25 \geq 0.05$ & $2.62 \geq 0.05$ & \\
\hline \multirow[t]{7}{*}{ Chlorantraniliprole } & 0 & Control & 17 & $22.1 \pm 7.7$ & - & \\
\hline & 2 & $0.05 x$ & 17 & $0.8 \pm 6.2$ & - & 1 \\
\hline & 4 & $0.10 \mathrm{x}$ & 17 & $6.8 \pm 1.4$ & - & 1 \\
\hline & 10 & $0.25 x$ & 17 & $2.1 \pm 6.5$ & - & 1 \\
\hline & 20 & $0.50 x$ & 17 & $0.0 \pm 0.0$ & - & 1 \\
\hline & 40 & $1 \mathrm{x}$ & 17 & $6.5 \pm 11.4$ & - & 1 \\
\hline & & $\chi^{2}, \mathrm{P}$ & & $2.69 \geq 0.05$ & - & \\
\hline \multirow{7}{*}{ Cyantraniliprole } & 0 & Control & 20 & $20.0 \pm 15.0 \mathrm{ab}$ & $25.0 \pm 15.3 \mathrm{a}$ & \\
\hline & 3.75 & $0.05 \mathrm{x}$ & 20 & $0.0 \pm 0.0 \mathrm{ab}$ & $0.0 \pm 0.0 \mathrm{a}$ & 1 \\
\hline & 7.50 & $0.10 \mathrm{x}$ & 20 & $0.0 \pm 0.0 \mathrm{a}$ & $0.0 \pm 0.0 \mathrm{a}$ & 1 \\
\hline & 18.75 & $0.25 x$ & 20 & $12.5 \pm 8.7 \mathrm{ab}$ & $15.6 \pm 8.8 \mathrm{ab}$ & 1 \\
\hline & 37.50 & $0.50 \mathrm{x}$ & 20 & $4.1 \pm 6.0 \mathrm{ab}$ & $13.3 \pm 15.3 \mathrm{ab}$ & 1 \\
\hline & 75.00 & $1 \mathrm{x}$ & 20 & $18.8 \pm 7.6 \mathrm{~b}$ & $35.6 \pm 6.0 \mathrm{~b}$ & 2 \\
\hline & & $\chi^{2}, \mathrm{P}$ & & $11.11 \leq 0.05$ & $15.78 \leq 0.05$ & \\
\hline
\end{tabular}

Values within columns followed by a different letter are significantly different according to Tukey's test ( $\mathrm{P} \leq 0.05)$. Values are mean $\pm \mathrm{SE}$; n: number of insects tested. Control not corrected by Abbott's formula.

IOBC class: 1 : Harmless (mortality $<30 \%)$, 2 : slightly harmful $(30 \leq$ mortality $\leq 79 \%)$, 3 : moderately harmful $(80 \leq$ mortality $\leq 99 \%)$, and 4: harmful (mortality $>99 \%$ ). 
Table 5. Mortality of adults of Cryptolaemus montrouzieri at 24 and $48 \mathrm{~h}$ after exposure to insecticide treatments on apple leaf discs.

\begin{tabular}{|c|c|c|c|c|c|c|}
\hline \multirow[b]{2}{*}{ Insecticide } & \multirow{2}{*}{$\begin{array}{l}\text { Active ingredient } \\
\text { concentration }\end{array}$} & \multirow{2}{*}{$\begin{array}{l}\text { Commercial } \\
\text { product rate }\end{array}$} & \multirow[b]{2}{*}{$\mathrm{n}$} & \multicolumn{2}{|c|}{ Corrected mortality } & \multirow{2}{*}{$\begin{array}{l}\text { IOBC } \\
\text { class }\end{array}$} \\
\hline & & & & $24 \mathrm{~h}$ & $48 \mathrm{~h}$ & \\
\hline & $\mathrm{mg} \mathrm{L}^{-1}$ & & & $\%$ & $\%$ & \\
\hline \multirow[t]{7}{*}{ Acetamiprid } & 0 & Control & 16 & $11.8 \pm 0.9 \mathrm{a}$ & $22.0 \pm 7.6 \mathrm{a}$ & \\
\hline & 0.42 & $0.05 \mathrm{x}$ & 16 & $15.7 \pm 7.8 \mathrm{a}$ & $48.3 \pm 9.9 b$ & 2 \\
\hline & 8.40 & $0.10 \mathrm{x}$ & 16 & $16.9 \pm 6.7 \mathrm{ab}$ & $41.4 \pm 17.4 \mathrm{~b}$ & 2 \\
\hline & 21.00 & $0.25 \mathrm{x}$ & 16 & $48.2 \pm 11.1 b c$ & $68.8 \pm 4.3 b$ & 2 \\
\hline & 42.00 & $0.50 x$ & 16 & $39.5 \pm 6.7 b$ & $60.3 \pm 17.4 b$ & 2 \\
\hline & 84.00 & $1 \mathrm{x}$ & 16 & $55.4 \pm 6.4 \mathrm{c}$ & $70.1 \pm 14.5 b$ & 2 \\
\hline & & $\chi^{2}, \mathrm{P}$ & & $45.5 \leq 0.01$ & $55.16 \leq 0.05$ & \\
\hline \multirow[t]{7}{*}{ Buprofezin } & 0 & Control & 15 & $6.7 \pm 3.8$ & $15.3 \pm 2.3$ & \\
\hline & 10 & $0.05 \mathrm{x}$ & 15 & $0.0 \pm 0.0$ & $0.0 \pm 0.0$ & 1 \\
\hline & 20 & $0.10 \mathrm{x}$ & 15 & $2.5 \pm 2.0$ & $0.0 \pm 0.0$ & 1 \\
\hline & 50 & $0.25 x$ & 15 & $2.5 \pm 2.0$ & $8.3 \pm 9.6$ & 1 \\
\hline & 100 & $0.50 \mathrm{x}$ & 15 & $0.0 \pm 0.0$ & $8.3 \pm 4.7$ & 1 \\
\hline & 200 & $1 \mathrm{x}$ & 15 & $7.1 \pm 3.8$ & $10.6 \pm 8.1$ & 1 \\
\hline & & $\chi^{2}, P$ & & $5.28 \geq 0.05$ & $6.19 \geq 0.05$ & \\
\hline \multirow[t]{7}{*}{ Pyriproxyfen } & 0 & Control & 15 & $11.3 \pm 8.0$ & $26.7 \pm 7.8$ & \\
\hline & 2.5 & $0.05 \mathrm{x}$ & 15 & $2.3 \pm 6.7$ & $0.0 \pm 0.0$ & 1 \\
\hline & 5.0 & $0.10 \mathrm{x}$ & 15 & $0.0 \pm 0.0$ & $0.0 \pm 0.0$ & 1 \\
\hline & 12.5 & $0.25 \mathrm{x}$ & 15 & $0.0 \pm 0.0$ & $0.0 \pm 0.0$ & 1 \\
\hline & 25.0 & $0.50 \mathrm{x}$ & 15 & $2.3 \pm 3.8$ & $0.0 \pm 0.0$ & 1 \\
\hline & 50.0 & $1 \mathrm{x}$ & 15 & $0.0 \pm 0.0$ & $0.0 \pm 0.0$ & 1 \\
\hline & & $\chi^{2}, \mathrm{P}$ & & $5.29 \geq 0.05$ & $3.08 \geq 0.05$ & \\
\hline \multirow[t]{7}{*}{ Chlorantraniliprole } & 0 & Control & 20 & $3.3 \pm 1.7$ & $10.0 \pm 2.9 \mathrm{a}$ & \\
\hline & 2 & $0.05 \mathrm{x}$ & 20 & $12.1 \pm 10.0$ & $16.7 \pm 15.3 \mathrm{ab}$ & 1 \\
\hline & 4 & $0.10 \mathrm{x}$ & 20 & $5.2 \pm 4.4$ & $20.4 \pm 7.3 \mathrm{ab}$ & 1 \\
\hline & 10 & $0.25 \mathrm{x}$ & 20 & $15.5 \pm 8.3$ & $24.1 \pm 11.7 \mathrm{~b}$ & 1 \\
\hline & 20 & $0.50 \mathrm{x}$ & 20 & $10.3 \pm 8.3$ & $18.5 \pm 9.3 \mathrm{a}$ & 1 \\
\hline & 40 & $1 \mathrm{x}$ & 20 & $15.5 \pm 7.3$ & $29.6 \pm 14.8 b$ & 1 \\
\hline & & $\chi^{2}, P$ & & $11.63 \leq 0.05$ & $15.37 \leq 0.01$ & \\
\hline \multirow[t]{7}{*}{ Cyantraniliprole } & 0 & Control & 20 & $1.7 \pm 1.7 \mathrm{a}$ & $5.0 \pm 2.9 \mathrm{a}$ & \\
\hline & 3.75 & $0.05 \mathrm{x}$ & 20 & $13.6 \pm 2.9 \mathrm{a}$ & $21.1 \pm 5.8 \mathrm{a}$ & 1 \\
\hline & 7.50 & $0.10 \mathrm{x}$ & 20 & $22.0 \pm 6.0 \mathrm{abc}$ & $42.1 \pm 11.6 \mathrm{ab}$ & 2 \\
\hline & 18.75 & $0.25 \mathrm{x}$ & 20 & $35.6 \pm 17.6 c$ & $64.9 \pm 14.5 b c$ & 2 \\
\hline & 37.50 & $0.50 x$ & 20 & $47.5 \pm 9.3 \mathrm{ab}$ & $73.7 \pm 12.6 \mathrm{~cd}$ & 2 \\
\hline & 75.00 & $1 \mathrm{x}$ & 20 & $40.7 \pm 10.9 c$ & $80.7 \pm 7.3 d$ & 3 \\
\hline & & $\chi^{2}, P$ & & $58.18 \leq 0.01$ & $131.56 \leq 0.01$ & \\
\hline
\end{tabular}

Values within columns followed by a different letter are significantly different according to Tukey's test $(\mathrm{P} \leq 0.05)$. Values are mean $\pm \mathrm{SE}$; n: number of insects tested. Control not corrected by Abbott's formula.

IOBC class: 1 : Harmless (mortality $<30 \%)$, 2: slightly harmful $(30 \leq$ mortality $\leq 79 \%)$, 3 : moderately harmful $(80 \leq$ mortality $\leq 99 \%)$, and

4: harmful (mortality $>99 \%$ ).

Both IGRs, buprofezin and pyriproxyfen, were harmless or slightly harmful to adults of several encyrtid parasitoid species of mealybugs (Wakgari and Giliomee, 2003; Rothwangl et al., 2004; Cloyd and Dickinson, 2006; Suma et al., 2009; Karmakar and Shera, 2018). However, when the field rate of buprofezin was applied on parasitized vine mealybug an increase in development time was found for Anagyrus sp. near pseudococci (Girault) and Coccidoxenoides perminutus (Timberlake) (Mgocheki and Addison, 2009). Our results agree with harmless or slightly harmful effects of both IGRs on adult A. flavidulus, which have been also described for A. flavidulus exposed to residues of 7 DAT in semi-field trials (Alvear et al., 2016).

For larvae and adults of C. montrouzieri pyriproxyfen and buprofezin were harmless (Cloyd and Dickinson, 2006; Alexander et al., 2013) which agree with our experimental results. However, other studies show that when pyriproxyfensprayed prey were used to fed larvae a slightly harmful effect was found (Planes et al., 2013; El Aalaoui et al., 2019). 
Chlorantraniliprole has been found harmless for A. mali (Gontijo, 2011; Mills et al., 2016), which agree with our results. We found chlorantraniliprole harmless at almost all concentrations (except for $0.25 \mathrm{x}$ ) and at the lower concentrations of cyantraniliprole on A. flavidulus. It seems that chlorantraniliprole could be safer than cyantraniliprole on A. flavidulus. For $C$. montrouzieri both diamides were mainly harmless or slightly harmful, with cyantraniliprole moderately harmful on adults at the minimum recommended rate.

It is important to emphasize that our results were from laboratory tests, which cannot be directly extrapolated to field conditions. Further bioassays should be performed to study the sublethal effects of those individuals who managed to survive this exposure and evaluate their effects on parasitism, predation, sex rate, clutch size, among others, in order to have a holistic view of the insecticide effects on natural enemies.

\section{CONCLUSIONS}

This study has determined the acute toxicity of various concentrations of six insecticides used in apple orchards on adults of Aphelinus mali and Acerophagus flavidulus and also on larvae and adults of Cryptolaemus montrouzieri. Neonicotinoids such as thiacloprid and acetamiprid were moderately harmful to harmful especially on the two parasitoids, and harmless and slightly harmful on larvae and adults of $C$. montrouzieri. Growth regulators such as buprofezin and pyriproxyfen, and the diamide chlorantraniliprole seem to be safe for adult parasitoids and for larvae and adults of $C$. montrouzieri. Finally, the newer diamide cyantraniliprole was less compatible than chlorantraniliprole with the natural enemies evaluated.

\section{ACKNOWLEDGEMENTS}

Thanks to XILEMA SpA for providing insects for bioassays. Also to CONICYT-ANID for Beca de Doctorado Nacional 2014 and CONICYT + PAI/Concurso Nacional de Tesis de Doctorado en el Sector Productivo 2015 + Folio 7815110001. Finally, thanks to Programa de Doctorado en Ciencias Agrarias, Universidad de Talca, for resources given to develop laboratory bioassays.

\section{REFERENCES}

Abbott, W.S. 1925. A method of computing the effectiveness of an insecticide. Journal of Economic Entomology 18:265-266. Ahumada, M.I., and Chorbadjian, R. 2019. Laboratory assays of the insecticidal activity of cyantraniliprole and imidacloprid on Brevicoryne brassicae, Myzus persicae (Hemiptera: Aphididae) and Trialeurodes vaporariorum (Hemiptera: Aleyrodidae) pests species and a biological control agent Chrysoperla defreitasi (Neuroptera: Chrysopidae). Chilean Journal of Agricultural Research 79:658-663.

Alexander, A., Krishnamoorthy, S.V., and Kuttalam, S. 2013. Toxicity of insecticides to the coccinellid predators, Cryptolaemus montrouzieri Mulsant and Scymnus coccivora Ayyar of papaya mealybug, Paracoccus marginatus Williams and Granara de Willink. Journal of Biological Control 27(1):18-23.

Alvear, A., Ripa, L., y Volosky, C. 2016. Efecto letal de residuos de plaguicidas sobre agentes de control biológicos de Pseudococcidae en pruebas de laboratorio y semi-campo. Abstract 61. In XXXVIII Congreso Nacional de Entomología, Talca, Chile. Sociedad Nacional de Entomología, Santiago, Chile.

Asante, S.K. 1997. Natural enemies of the woolly apple aphid, Eriosoma lanigerum (Hausmann) (Hemiptera: Aphididae): A review of the world literature. Plant Protection Quarterly 12(4):166-172.

Beers, E.H., Horton, D.R., and Miliczky, E. 2016. Pesticides used against Cydia pomonella disrupt biological control of secondary pests of apple. Biological Control 102:35-43.

Beers, E.H., Martinez-Rocha, L., Talley, R.R., and Dunley, J.E. 2009. Lethal, sublethal, and behavioral effects of sulfurcontaining products in bioassays of three species of orchard mites. Journal of Economic Entomology 102(1):324-335.

Casida, J.E. 2018. Neonicotinoids and other insect nicotinic receptor competitive modulators: progress and prospects. Annual Review of Entomology 63:125-144.

Charles, J.G. 2011. Using parasitoids to infer a native range for the obscure mealybug, Pseudococcus viburni, in South America. BioControl 56(2):155-161.

Cloyd, R.A., and Dickinson, A. 2006. Effect of insecticides on mealybug destroyer (Coleoptera: Coccinellidae) and parasitoid Leptomastix dactylopii (Hymenoptera: Encyrtidae), natural enemies of citrus mealybug (Homoptera: Pseudococcidae). Journal of Economic Entomology 99(5):1596-1604. 
Cross, J.V., Solomon, M.G., Babandreier, D., Blommers, L., Easterbrook, M.A., Jay, C.N., et al. 1999. Biocontrol of pests of apples and pears in northern and central Europe: 2. Parasitoids. Biocontrol Science and Technology 9(3):277-314.

Dumaniya, S.G., Patel, M., and Siddhapara, M.R. 2015. Toxicity of insecticides to Cryptolaemus montrouzieri (Mulsant). Journal of Cotton Research and Development 29(1):121-124.

Ebadollahi, A., and Sadeghi, R. 2020. Comparison of the toxicity and repellency of two conventional neonicotinoids and a coconut-derived insecticide soap toward the parasitoid wasp Aphelinus mali Haldeman, 1851. Acta Agriculturae Slovenica 115(1):97-103.

El Aalaoui, M., Bouharroud, R., Sbaghi, M., El Bouhssini, M., Hilali, L., Dari, K., et al. 2019. Comparative toxicity of different chemical and biological insecticides against the scale insect Dactylopius opuntiae and their side effects on the predator Cryptolaemus montrouzieri. Archives of Phytopathology and Plant Protection 52(1-2):155-169.

Gontijo, L.M. 2011. Integrated biological control of woolly apple aphid in Washington. PhD Dissertation. Washington State University, Pullman, Washington, USA. Available at https://research.libraries.wsu.edu:8443/xmlui/bitstream/ handle/2376/3523/Gontijo_wsu_0251E_10331.pdf?sequence=1\&isAllowed=y (accesed January 2020).

González, R.H. 2010. Pseudocóccidos de importancia frutícola en Chile. Universidad de Chile, Santiago, Chile.

Howard, L.O. 1929. Aphelinus mali and its travels. Annals of the Entomological Society of America 12(3):341-368.

Jamil, R.Z.R., Vandervoort, C., and Wise, J.C. 2019. Residual toxicity of insecticides to Neoseiulus fallacis (Acari: Phytoseiidae) in apples. Journal of Economic Entomology 112(5):2262-2267.

Jones, V.P., Unruh, T.R., Horton, D.R., Mills, N.J., Brunner, J.F., Beers, E.H., et al. 2009. Tree Fruit IPM programs in the western United States: the challenge of enhancing biological control through intensive management. Pest Management Science 65(12):1305-1310

Jong, P., and van Alphen, J. 1989. Host size selection and sex allocation in Leptomastix dactylopii, a parasitoid of Planococcus citri. Entomologia Experimentalis et Applicata 50:161-169.

Kairo, M., Paraiso, O., Gautam, R., and Peterkin, D. 2013. Cryptolaemus montrouzieri (Mulsant) (Coccinellidae: Scymninae): a review of biology, ecology, and use in biological control with particular reference to potential impact on non-target organisms. CAB Reviews: Perspectives in Agriculture, Veterinary Science, Nutrition and Natural Resources 8(005):1-20.

Karamaouna, F., and Copland, M.J.W. 2000. Host suitability, quality and host size preference of Leptomastix epona and Pseudaphycus flavidulus, two endoparasitoids of the mealybug Pseudococcus viburni, and host size effect on parasitoid sex ratio and clutch size. Entomologia Experimentalis et Applicata 96:149-158.

Karmakar,P., and Shera, P.S. 2018. Lethal and sublethal effects of insecticides used in cotton crop on the mealybug endoparasitoid Aenasius arizonensis. International Journal of Pest Management 66(1):13-22.

Lahm, G.P., Selby, T.P., Freudenberger, J.H., Stevenson, T.M., Myers, B.J., Seburyamo, G., et al. 2005. Insecticidal anthranilic diamides: A new class of potent ryanodine receptor activators. Bioorganic and Medicinal Chemistry Letters 15(22):4898-4906.

Lahm, G.P., Stevenson, T.M., Selby, T.P., Freudenberger, J.H., Cordova, D., Flexner, L., et al. 2007. Rynaxypyr ${ }^{\mathrm{TM}}$ : A new insecticidal anthranilic diamide that acts as a potent and selective ryanodine receptor activator. Bioorganic and Medicinal Chemistry Letters 17(22):6274-6279.

Mani, M. 2018. Hundred and sixty years of Australian lady bird beetle Cryptolaemus montrouzieri Mulsant- a global view. Biocontrol Science and Technology 28(10):938-952.

Mgocheki, N., and Addison, P. 2009. Effect of contact pesticides on vine mealybug parasitoids, Anagyrus sp. near pseudococci (Girault) and Coccidoxenoides perminutus (Timberlake) (Hymenoptera: Encyrtidae). South African Journal for Enology and Viticulture 30(2):110-116.

Mills, N.J., Beers, E.H., Shearer, P.W., Unruh, T.R., and Amarasekare, K.G. 2016. Comparative analysis of pesticide effects on natural enemies in western orchards: A synthesis of laboratory bioassay data. Biological Control 102:17-25.

Noyes, J.S. 2003. Universal Chalcidoidea Database. Natural History Museum, London, UK. Available at https://www.nhm.ac.uk/our-science/data/chalcidoids/database/countries.dsml (accessed September 2020).

Planes, L., Catalán, J., Tena, A., Porcuna, J.L., Jacas, J.A., Izquierdo, J., et al. 2013. Lethal and sublethal effects of spirotetramat on the mealybug destroyer, Cryptolaemus montrouzieri. Journal of Pest Science 86(2):321-327.

Rogers, D.J., Lo, P.L., and Walker, J.T.S. 2015. Vulnerability of pest management in New Zealand apples: pesticide toxicity to Aphelinus mali (Hymenoptera: Encyrtidae). New Zealand Plant Protection 68:299-305.

Rogers, D.J., Sharma, N., Stretton, D., and Walker, J.T.S. 2011. Toxicity of pesticides to Aphelinus mali, the parasitoid of woolly apple aphid. New Zealand Plant Protection 64:235-240.

Rothwangl, K.B., Cloyd, R.A., and Wiedenmann, R.N. 2004. Effects of insect growth regulators on citrus mealybug parasitoid Leptomastix dactylopii (Hymenoptera: Encyrtidae). Journal of Economic Entomology 97(4):1239-1244.

Selby, T.P., Lahm, G.P., Stevenson, T.M., Hughes, K.A., Cordova, D., Annan, B., et al. 2013. Discovery of cyantraniliprole, a potent and selective anthranilic diamide ryanodine receptor activator with cross-spectrum insecticidal activity. Bioorganic and Medicinal Chemistry Letters 23(23):6341-6345.

Sterk, G., Hassan, S.A., Baillod, M., Bakker, F., and Bigler, F. 1999. Results of the seventh joint pesticide testing programme carried out by the IOBC/WPRS-Working Group 'Pesticides and Beneficial Organisms'. Biocontrol (44):99-117. 
Suma, P., Zappalà, L., Mazzeo, G., and Siscaro, G. 2009. Lethal and sub-lethal effects of insecticides on natural enemies of citrus scale pests. BioControl 54(5):651-661.

Tunaz, H., and Uygun, N. 2004. Insect growth regulators for insect pest control. Turkish Journal of Agriculture and Forestry 28(6):377-387.

Wakgari, W.M., and Giliomee, J.H. 2003. Natural enemies of three mealybug species (Hemiptera: Pseudococcidae) found on citrus and effects of some insecticides on the mealybug parasitoid Coccidoxenoides peregrinus (Hymenoptera: Encyrtidae) in South Africa. Bulletin of Entomological Research 93(3):243-254.

Wang, Z., Dai, P., Yang, X., Ruan, C., Biondi, A., Desneux, N., et al. 2019. Selectivity of novel and traditional insecticides used for management of whiteflies on the parasitoid Encarsia formosa. Pest Management Science 75(10):2716-2724.

Weddle, P.W., Welter, S.C., and Thomson, D. 2009. History of IPM in California pears - 50 years of pesticide use and the transition to biologically intensive IPM. Pest Management Science 65(12):1287-1292. 